\title{
Two Layers Approach for Website Testing
}

\author{
Sether Riaz \\ Institute of Information and Communication Technology, \\ University of Sindh, Jamshoro
}

\begin{abstract}
Websites have become major attention to users who prefer Internet knowledge that save time and reduce human efforts, and to offer error free websites to the users, this study divides the testing process into two layers approach by which testers will be able to find which tests are to run first on a website. This study uses literature to understand the nature of tests and on what part of the websites tests are run and divides them. This study collects seven tests and divides them on the basis of two layers which are concentrating on the principals of main services and associative services.
\end{abstract}

\section{Keywords}

Website testing, Main services, Associative services, two layers,

\section{INTRODUCTION}

Web development is a challenging and complex process with larger number of components interacting heterogeneously. Although websites construction has been evolved to some discipline but there is still lack on the engineering approach for building a web system and the engineering process itself is still to be engineered (1). In the year 2000, Paul Gerrard gave twenty testing techniques that summarized the testing procedure into categories (2). After Gerrard's report on testing there were occasions when testing procedure was added with new tests to make testing process reliable and with respect to the technological need. Following Gerrard approach in 2012, a paper reported challenges that websites development face and suggested a testing plan to tackle those challenges (3). In year 2012, a paper gave a test plan and suggested that how these test plan can help software development (4). There were various occasions when experts added new research in website testing, consider a paper in 2013 which gave highlights on genetic algorithm and various problems that software testing faces (5). In year 2013 a paper discussed the approach on functionality testing, suggested a test plan and indicated that the mentioned methods will help fully test a website both on user and server end (6). Keeping the website testing in consideration, a paper in 2014 describes the testing process with the help of automation and manual testing, and suggested techniques to test a website using automation testing (7). A paper on performance testing gave methodology and testing plan to reduce website failures (8). Looking at these papers author can say that testing process is constantly evolving as the researchers are developing new and latest techniques to best test websites. These test techniques include but not limited to the program and application execution with intentions to finding software bugs (9). While more tests and procedures are developed to make testing sophisticated, the need to develop a model where these tests can be divided is also increasing. Consider Gerrard's research where Gerrard suggests twenty techniques for testing websites, but those and various others tests need a model where testing can be run on the basis of their nature and priority. For making this possible, it is necessary to understand that, which tests are to be run first, which test run on main services and which tests are conducted on associative services. This understanding of the testing process will help web development and categorize the tests so that a better website can be made to satisfy the user visit.

\section{METHODS AND MATERIAL}

Websites have become common these days, business and sales rely on them for delivering necessary information so that they can increase business potential, awareness and achieve targets. With this rapid change in need of websites, it is crucial to test them for error identification. While testing websites, a tester must be aware of different type of tests that run on websites for error identification but consider the fact that there are many tests and finding those tests which are crucial and needed to be run first so that a reliable website can be produce is difficult. For making testing process more sophisticated, author divides testing in two layers, and both of the layers concentrate on categorizing the tests based on their nature. By using approach that is mentioned in this research will help with followings.

- $\quad$ There are different tests mixed together and finding those tests which are crucial to run will help testers to summarize testing into two types and by doing that testers will be able to focus on necessary tests that are linked with main services and then move towards conducting less necessary tests which are associated with the services.

- This study will help in finding the nature of different tests with the help of literature that is available in the field of website testing.

- The study will divide the testing design based on layer based approach for besting the testing process.

To fulfill these objectives, it is necessary to understand the difference of website's main services and associative services so that testing can be divided on the basis of their nature.

\subsection{Main Services}

A main service in a website is anything that user interact with and accesses it. There are various main services available as tabs, buttons, links, context and other data that websites offer to the users. In this study, author demonstrates with the help of literature to organize and divide tests on the basis of their nature. In this research, those tests which are run on main services of websites are referred as layer one tests. These layer one tests check main services of websites for ensuring they are error free.

\subsection{Associate Services}

A service that is not part of main web frame but play important part in providing a user friendly website are referred as associative services. Four of the associate service tests that are mention in this article are navigation, browser, host response and performance. These associative services are not visibly as main services to the user but play important part in making a website user friendly. Those tests which are run on associative services are referred as layer two tests. These 
tests check associate services and ensure that website is user friendly.

\section{SOLUTION TO THE PROBLEM}

After series of literature review, author found different tests that can be run on websites for error identification, and divides those tests on the basis of their nature. In this study author collected seven type of tests and organized them using two layers approach. The two layers approach is a theoretical model by which testing system is divided into two layers. The first layer is referred as layer one and the second layer is referred as layer two. Both of the layers concentrate on type of test and divides the test according to its nature.

For dividing different type of tests, author selected seven tests which are mentioned below.

- Functionality test

- Browser compatibility test

- Contextual test

- Host response test

- $\quad$ Navigation test

- Performance test

- $\quad$ Field test

These are the seven type of tests that author selected to divide them. For diving the tests according to their nature, testers need to answer one question that will help decide the nature of test and prioritize it. The question that need to answer is "whether test is run on the main service or associative service?" And if the test is run on the main service then it is important to conduct it first and then move towards layer two tests.

\subsection{Layer One Tests}

Layer one tests is a conceptual model where author puts different type of tests together on the basis of their nature. These tests are divided with keeping two concepts in consideration. The concepts are "whether test that is run on the website is checking services or it is checking associative services". The tests which are run to check main services are referred as layer one tests and further understanding of the model that author constructed is explain under.

\subsubsection{Functionality Test}

A paper describes the functionality as test of links, forums, database connections, test of internal links and all the links that are present in the website (3). Keeping the point, author can say that functionality test is about checking of links that are available in a website. If running the question that author suggested must be answer to understand the nature of test then functionality test is about testing of main services i.e. links that are part of the website so author put this test in layer one category.

\subsubsection{Contextual Test}

A paper describes the contextual testing as checking of grammar, spelling mistakes, completeness of content in web pages (2). With above definition, author can say that context testing is about testing of context that is available in a web page. Author considers context as a main service that web pages provide and divided this type of test in the category of layer one tests which are testing main services of websites.

\subsubsection{Field Test}

Author refers field test as checking of different fields in websites. A paper explains that testing of search options and similar entity is necessary to validate websites (3). A research indicates that specific area testing is necessary during testing of website (4). If author keeps both of these points in consideration then field testing is about checking of fields that are available for the users including search bar, user account fields and other similar fields. If looking at the nature of this test then field test is run on main services of a website so author categorized it in layer one tests.

\subsection{Layer Two Tests}

Layer two tests is a model in which author categorizes those tests which are run on associative services. These tests were put in this category on the basis of their nature. There are many tests which can be categorized using layer two principal and most of these tests are run to enhance the user experience or on particular service testing. As compare to layer one, these tests have less importance because first thing that testers should focus on is to improve the main services and if the main services are working fine then moving towards layer two tests is a good strategy than by conducting random tests on the websites.

\subsubsection{Browser Compatibility Test}

Browser compatibility test concerns with checking of whether content, pages and information is loaded in browser correctly (4). Keeping the point that browser testing concerns with testing of whether the main services are working fine with the browser or not, author concluded that browser compatibility testing is about testing of associative services, which means browser test comes in the category of layer 2 tests.

\subsubsection{Navigation Test}

Navigation concern with the use of a webpage i.e. user moves from one page to another, visits links, click on images, etc. (10). If author look at the nature of this test then navigation is about checking of how user moves from links to links. By the definition provided by miller's paper, author categorized this test in layer two tests category.

\subsubsection{Host Response Test}

Host response concerns with how fast website responses to the user, whether user moves on or gives up (10). If running the question whether testing is done on the main service or associative service then author easily can say that host response is not a main service that websites offer, it's an associative service and author has categorized all the associative service tests in layer two.

\subsubsection{Performance Test}

A research on performance testing says that performance testing is run to accomplish Assess production readiness, evaluate against performance criteria, Compare performance characteristics of multiple systems or system configurations, Find the source of performance problems, Support system tuning, and Find throughput levels (8). Keeping the point that if performance testing is based on how well system is performing then performance test can be categorized into layer two tests.

\section{SIGNIFICIENCE OF THE STUDY}

Web applications have been deployed at very fast pace and have help a fast adaptation, but at the same time there has been decreased in quality of software. Therefore, all of the website entities must be tested to make web applications successfully adopted. Testing must be flexible and able to 
handle their dynamic nature (8). Website testing ensures that links are not broken, no misspelled words, no graphic issues, no bugs in the software and downtime is specified (6). Keeping the process in consideration that most of the testing process involves testing of websites by keeping the number of tests that makes difficult to recognized which tests are to be run initially and which tests can be run later, so making the process of testing more sophisticated where testers will be able to recognize tests on the basis of test's nature. This study will help organizations to focus on delivering quality websites by realizing that most important part of the website is main services so testing using tests which are run on main services is a good approach to take. This will ensure that websites main services are working fine or not, and if they are fine then testers can move toward layer two for testing associative services for a quality website.

\section{CONCLUSION}

This study was conducted keeping the website testing processes in consideration and making them sophisticated where testers will be able to test websites using two layers approach that is mentioned in this paper for better testing. There are various occasions when testers are unable to understand testing of websites and because of the random testing process, the websites quality is compensated, but for making the process sophisticated it is necessary to follow a step by step approach where testing can be done by keeping websites perspective. There are different websites available and by keeping the websites perspective in consideration means a tester need to make certain that, what are the main services website is offering and then test them on the basis of main services and associative services. The next stage of the research will be to carry out study based on theoretical model that is mentioned in this paper, to ensure the model can be applied to the testing process, it is necessary to collect more tests in future and summarize them with the help of techniques which are mentioned in this paper, and using this model conducting practical testing of websites to ensure that model works on the basis of theory that is mentioned in this paper.

\section{ACKNOWLEDGEMENT}

Author want to thank his parents for making him eligible to conduct this study and supporting in all means of life. Without the help of his parents, this article or any of author's study would not have been possible. Author want to give all the credit of this study to his parents and want to thank them for all the support they gave, which enabled author to complete this paper.

\section{REFERENCES}

[1] D. I. Zahran, H. A. Al-Nuaim, M. J. Rutter and D. Benyon, "A comparative approach to web evaluation and website evaluation methods," International Journal of Public Information Systems, vol. 1, pp. 21-39, 2014.

[2] Paul Gerrard, "Risk-Based E-Business Testing," Systeme Evolutif Ltd, 2000

[3] S. Kundu, "Web Testing: Tool, Challenges and Methods," International Journal of Computer Science, vol. 9, no. 2, pp. 481-486, 2012.

[4] D. Gupta, "Load testing of websites," International Journal of Latest Trends in Engineering and Technology, vol. 1, no. 3, pp. 91-97, 2012.

[5] C. Sharma, S. Sabharwal and R. Sibal, "A Survey on Software Testing Techniques using Genetic Algorithm," International Journal of Computer Science, vol. 10, no. 1, pp. 381-393, 2013.

[6] M. S. Hussain1, A. Ali2 and J. Shafi, "Enhance Websites Testing via Functional and Non-Functional Approach: Case Study," International Journal of Application or Innovation in Engineering \& Management, vol. 2, no. 5, pp. 66-72, 2013.

[7] M. Sharma and R. Angmo, "Web based Automation Testing and Tools," International Journal of Computer Science and Information Technologies, pp. 908-912, 2014.

[8] V. Hegde and P. M. S, "Web Performance Testing: Methodologies, Tools and Challenges," International Journal of Scientific Engineering and Research, vol. 2, no. 1 , pp. 67-73, 2014

[9] T.Amruthavalli, S.MahaLAkshmi and K.HariKrishnan, "A Review On Software Testing In SDIC And Testing Tools," International Journal Of Engineering And Computer Science, vol. 3, no. 9, pp. 8188-8197, 2014.

[10] E. Miller, "Website testing," Software Research, Inc, 2000. 